\title{
Cuidados paliativos: por detrás dos olhos de um estudante
}

Miguel Julião*

\section{RESUMO}

No âmbito dos Cuidados Paliativos, a Medicina Paliativa é um conhecimento médico, científico, rigoroso e humanizado que trata o doente e a sua família nas diversas fases de uma doença crónica, progressiva e incurável.

Durante o seu percurso académico, o estudante de Medicina centraliza o seu esforço no estudo biomédico aprofundado dos processos fisiopatológicos, do diagnóstico e terapêutica dirigida a doenças específicas. O currículo médico pré-graduado não contempla o ensino obrigatório de Cuidados Paliativos e raramente refere o indivíduo na sua vertente holística, embora existam recomendações, como é exemplo o texto intitulado «O Licenciado Médico em Portugal: Core Graduates Learning Outcomes Project» desenvolvido pela Faculdade de Medicina de Lisboa.' Os jovens estudantes de Medicina são treinados predominantemente como cientistas biomédicos, incentivados a tornarem-se médicos de cura de doença, mais do que healers/cuidadores do doente como um todo.

É desejável que a formação específica pré-graduada no âmbito de Cuidados Paliativos penetre lentamente a estrutura académica para que, idealmente no futuro, faça parte obrigatória do currículo médico, como aliás figura nas orientações da European Association of Palliative Care.

Até que tal aconteça o autor, através da sua experiência pessoal, pretende fornecer algumas linhas orientadoras para ultrapassar os obstáculos apresentados ao jovem estudante de Medicina quando confrontado com a necessidade de transmitir e colocar em prática os princípios dos Cuidados Paliativos no seio das equipas médicas que integra e que não possuem conhecimento nesta área específica.

Palavras-Chave: Cuidados Paliativos; Ensino Médico Pré-Graduado.

\section{INTRODUÇÃO}

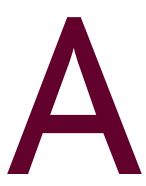
Medicina tem sido tradicionalmente considerada como uma profissão de cura e a Medicina moderna legitimou cada vez mais este processo através do conhecimento científico.

$\mathrm{O}$ «casamento» entre Medicina e Ciência permitiu aos médicos uma intervenção activa na história natural da doença, através da terapêutica, da prevenção e erradicação de eventos desfavoráveis. No auge deste sucesso, o estudante é treinado numa sólida raiz biomédica e foca o seu estudo em três componentes essenciais: diagnóstico, tratamento e prevenção. ${ }^{3}$

Grandes mudanças têm ocorrido nos padrões de morbilidade e mortalidade com os quais a Medicina se

*Interno do Internato Complementar de Medicina Geral e Familiar, Centro de Saúde de Marvila

Consultor Científico do Centro de Estudos de Medicina Baseada na Evidência, Faculdade de Medicina de Lisboa

Colaborador voluntário da Unidade de Cuidados Continuados e Paliativos do Hospital da Luz confronta.

Muita da necessidade actual de cuidados médicos especializados surge do crescente número de doenças crónicas, progressivas e incuráveis. Perante esta realidade, a prevenção pode ser tão importante como o tratamento curativo e/ou paliativo e os cuidados globais ao doente e à sua família tão importantes como a cura.

Em 2002, a OMS definiu Cuidados Paliativos como uma abordagem científica, rigorosa e humanizada que visa melhorar a qualidade de vida dos doentes que enfrentam problemas decorrentes de uma doença incurável e/ou grave, com prognóstico limitado, através da prevenção e alívio do sofrimento, com recurso à identificação precoce e tratamento rigoroso dos problemas não só físicos como a dor, mas também psicossociais e espirituais.

Nesta linha de raciocínio, muitos autores têm sublinhado a necessidade de healing rather than curing, ou seja, numa adaptação possível para o português, cui- 
dar ou recuperar mais do que curar. Eric Cassel avança até que se pode cuidar ou recuperar uma pessoa, sendo completo neste processo, sem que se altere sigificativamente a doença orgânica de base. ${ }^{4}$

Assim, actualmente, doença assume uma perpectiva mais abrangente: a questão não se coloca em tratar ou não tratar mas sim determinar qual a actuação mais apropriada em função das perspectivas biológicas e das circunstâncias pessoais e sociais do doente. Será este, em conjunto com tantos outros, um novo e desafiante conhecimento a ser leccionado nas Faculdades de Medicina.

Este crescente avanço da Medicina incrementa modificações constantes no currículo médico.

Os princípios e o ensino de Cuidados Paliativos estão a ser lentamente introduzidos no currículo médico pré-graduado.

Glare e Virik concluem que as escolas médicas australianas enfatizam a fisiopatologia da doença e a terapêutica mais do que a abordagem holística dos Cuidados Paliativos. ${ }^{5}$

Billings e Block avançam que a formação em Cuidados Paliativos é inadequada em anos clínicos e, quando alertados para a existência da mesma, os estudantes apresentam uma adesão favorável. ${ }^{6}$

Outros autores, como Dowling e Broomfield, referem que a identificação de formadores é difícil, existindo uma desresponsabilização académica no ensino desta área. As rotações de Cuidados Paliativos oferecidas aos estudantes têm uma carga horária reduzida e o seu conteúdo é predominantemente teórico. ${ }^{7}$

Os estudantes de Medicina em todo o mundo centralizam o seu esforço no estudo biomédico mais aprofundado dos processos fisiopatológicos, da terapêutica dirigida a doenças específicas. São treinados como cientistas biomédicos, incentivados a tornarem-se médicos de cura de doença, mais do que cuidadores do doente como um todo.

O currículo médico mais ancestral, baseado na ideia de médico como healer ou curador/cuidador, passou assim a ser ultrapassado pela pressão da Medicina tecnológica alicerçada em múltiplos meios complementares de diagnóstico, existindo poucos momentos de educação médica centrados na perspectiva holística do indivíduo ou mesmo em áreas como comunicação, cuidados e terapêutica específica em final de vida.
A recuperação no sentido holístico desvaneceu-se da estrutura académica, desapareceu do currículo médico pré-graduado e raramente é discutida na literatura médica.

O preenchimento curricular excessivo é apontado usualmente como justificação para a exclusão de Cuidados Paliativos como parte integrante da educação médica. O objectivo das Faculdades de Medicina é o de formar médicos com capacidade de actualização permanente, com excelente actividade assistencial, adaptabilidade e capacidade de trabalhar em equipa, promovendo um trabalho de excelência na saúde de todos os indivíduos em todas as suas fases de vida.

A introdução desta área do conhecimento médico no currículo pré-graduado é premente considerando o número crescente de doenças crónicas e degenerativas. Contudo, é limitada a evidência científica que poderá guiar a introdução do ensino pré-graduado de Cuidados Paliativos.

Algumas escolas médicas já introduziram os Cuidados Paliativos no seu currículo pré-graduado. A Harvard Medical School conta com um programa de ensino de Medicina Paliativa desde o $1^{\circ}$ ano, leccionando temas como princípios gerais dos Cuidados Paliativos, tratamento de dor e outros sintomas, cuidados activos ao doente terminal, luto, comunicação e especificidade do doente geriátrico. As suas aulas servem-se não só das apresentações dos palestrantes, mas também de discussão de casos clínicos ou de apresentações feitas por doentes.

No mesmo sentido, a Faculdade de Medicina de Lisboa, através do Centro de Bioética e do Núcleo de Cuidados Paliativos, criou a cadeira optativa de Cuidados Terminais, contendo no seu programa áreas como filosofia dos Cuidados Paliativos, controlo sintomático (dor, dispneia, náuseas, vómitos e outros), apoio à família, apoio no luto, trabalho em equipa, cuidados específicos na fase agónica, comunicação (role-playing para treino de gestão de más notícias) e principais problemas éticos no fim de vida.

Este artigo de opinião surge naturalmente de uma vivência, de um percurso pessoal na área da Medicina Paliativa. Assim, faz sentido que fale sucintamente da minha experiência, mostrando os seus momentos mais marcantes e tentando justificar a opinião acerca desta área médica prioritária. O primeiro grande marco deu- 
se ainda enquanto estudante do $4^{\circ}$ ano do Curso de Medicina, através da cadeira opcional de Cuidados Paliativos. Foi após a primeira aula que se levantou em mim uma surpresa incrível: a de uma Medicina nova, completa, científica e humanizada. De tal forma foi este feliz «encontro» que decidi que tinha encontrado «a área médica que queria exercer até ao final da minha carreira». Ao longo do resto do Curso tive a oportunidade de realizar intervenções práticas junto dos doentes e suas famílias (por exemplo a introdução de nova via de administração de soros e fármacos, como a via subcutânea, ignorada até então) nos diversos Serviços de Medicina Interna e Cirurgia por onde passei. Foram crescendo as solicitações por parte das equipas médicas de apresentações acerca desta nova temática. Já recém-licenciado, seguiu-se a formação pós-graduada da Faculdade de Medicina de Lisboa em Cuidados Terminais, após o que integrei durante cerca de 2 anos a Equipa de Cuidados Paliativos da Casa de Saúde da Idanha. Actualmente lecciono com grande alegria e entusiasmo alguns módulos da cadeira opcional de Cuidados Paliativos da Faculdade de Medicina de Lisboa e encontro-me a trabalhar como colaborador voluntário do Serviço de Cuidados Paliativos e Continuados do Hospital da Luz, podendo contactar com a realidade da Medicina Paliativa e trabalhando em alguns projectos de investigação. Ao longo de todo este período, os momentos de pesquisa e formação (nacional e internacional) foram constantes, pilares necessários a uma actualização e conhecimento, garantes essenciais de uma medicina de qualidade que visa garantir o melhor tratamento e apoio aos doentes e suas famílias.

A constante reflexão pessoal acerca desta área enquanto estudante de Medicina e médico, uma pesquisa bibliográfica acerca do ensino pré-graduado e Cuidados Paliativos (entre o $4^{\circ}$ ano do curso e o ano de 2007), as dificuldades que se apresentam ao nível pessoal e enquanto elemento integrante de uma equipa médica sem formação paliativa específica, justificam este trabalho onde se pretende fornecer aos jovens estudantes linhas orientadoras sucintas que possam ajudar a ultrapassar alguns obstáculos que surgem entre o estudante, a necessidade de uma prática clínica baseada em evidência científica aplicada ao doente, à sua família, ao seu sofrimento e ao trabalho em equipa.

\section{SER ESTUDANTE}

Por estudante entende-se aquele que estuda, o aluno, o discípulo, o colegial.

Estudar é, assim, por definição, aquisição de novas noções, examinar, decorar, aprender.

Enquanto estudante, o indivíduo possui reduzido conhecimento médico geral e falta-lhe autonomia e capacidade de decisão. A prática clínica é ainda um exercício de medicina baseada na experiência do mestre que ensina, mais do que um exercício de medicina baseada em evidência.

A necessidade de obtenção de uma classificação em determinada área académica, juntamente com todos os factores anteriormente referidos, coloca o estudante numa posição desfavorável quando se trata de introduzir novos conhecimentos no seio do grupo de trabalho.

Não existindo formação obrigatória em Cuidados Paliativos, o aluno que a possui vê dificultada a partilha do conhecimento com os seus pares de trabalho.

Contudo, o estudante é também considerado motor de mudança, indivíduo jovem, em vantagem na obtenção de informação médica credível e, por isso, capaz de transmissão de uma medicina actualizada (Figura 1).

\section{A EQUIPA MÉDICA SEM FORMAÇÃO ESPECÍFICA EM CUIDADOS PALIATIVOS}

É constante a afirmação de que «Cuidados Paliativos são uma área transversal a qualquer especialidade médica».

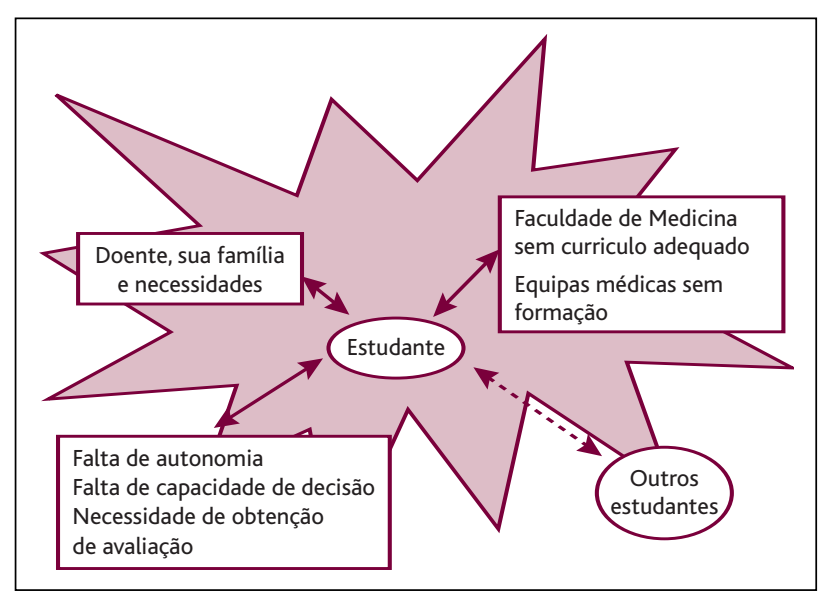

Figura 1. O estudante de Medicina no centro de uma relação enevoada 
Muitos médicos afirmam que existe pouca especificidade de tratamento em final de vida, ou mesmo, poucas opções terapêuticas dirigidas à sintomatologia das doenças crónicas, progressivas e incuráveis.

$\mathrm{O}$ afastamento e o desconhecimento da maioria dos profissionais de saúde acerca de questões como lidar com a morte, transmissão de más notícias, técnicas de comunicação adequadas com a família e a equipa, controlo sintomático e terapêutica farmacológica específica e adequada leva a que o estudante sinta resistência à introdução de um «novo» conhecimento médico, científico, rigoroso e humanizado.

As equipas médicas formadas para a cura da doença orgânica actuam com frequência através da futilidade diagnóstica e terapêutica e vivem a situação evolutiva e irreversível do doente como um insucesso pessoal.

Não conhecer, por exemplo, sinais e sintomas do doente agónico, ou mesmo as terapêuticas específicas para o final de vida, cria no seio das equipas médicas uma sensação de medo e insegurança face à constatação da necessidade de aperfeiçoamento pessoal, profissional e, de forma mais lata, ético.

\section{COMO ULTRAPASSAR ALGUNS OBSTÁCULOS}

- PERSEVERANÇA E DETERMINAÇÃO

- ORGANIZAÇÃO DE BIBLIOGRAFIA PRÓPRIA (a título exemplificativo)

LITERATURA CIENTÍFICA

- Artigos científicos

- Livros de texto - Cuidados Paliativos ${ }^{8}$ Manual de Cuidados Paliativos ${ }^{9}$ Oxford Textbook of Palliative Medicine $^{10}$

- Internet-http://www.palliativedrugs.com http://www.apcp.com.pt http://www.aahpm.com

LITERATURA NÃO-CIENTÍFICA

Ex.: Às Terças com Morrie ${ }^{11}$

Morrie in his own Words ${ }^{12}$

Óscar e a Senhora Cor-de-Rosa ${ }^{13}$

A Última Aula ${ }^{14}$

- FORMAÇÃO E ACTUALIZAÇÃO CONSTANTES

- CERTEZA E CONFIANÇA NO QUE SE AFIRMA E PRATICA

A necessidade de decisões clínicas tomadas de forma sólida e integrada deve basear-se na melhor evi- dência científica disponível, para que se possa melhorar a actividade assistencial ao indivíduo, à sua família e, numa perspectiva mais alargada, à sociedade. É inúmera a investigação de alta qualidade disponível na Medicina Paliativa, para que se possam fundamentar as nossas escolhas.

Na maioria das situações deveremos ser capazes de estabelecer o seguinte diálogo:

- «Em que se baseia?»

- «Em evidência científica.»

- PARTILHA DE EXPERIÊNCIAS COM OS PARES DE TRABALHO E A EXPERIÊNCIA DO TRABALHO EM EQUIPA

Os problemas complexos encontrados na Medicina Paliativa exigem um esforço comum, partilhado e uníssono. Este esforço baseia-se numa abordagem transdisciplinar, onde cada elemento da equipa constrói uma concepção comum cuja finalidade última é o bem-estar do doente e da sua família.

O estudante deve entender o trabalho articulado em equipa como garante essencial para o sucesso terapêutico. É nesta fase académica que poderá aprender e aplicar na sua prática clínica futura princípios essenciais como as relações profissionais de multidisciplinaridade, a aprendizagem em equipa e a noção de liderança. - ORGANIZAR OBJECTIVOS: «SEMANAS PESSOAIS DE CUIDADOS PALIATIVOS» (a título exemplificativo)

O estudante pode calendarizar semanas com objectivos pessoais claros que considera relevantes para o desenvolvimento do seu conhecimento e prática em Cuidados Paliativos. Assim, mais do que preocupado com a aprendizagem global, divide-a em temas e tenta observar os seus aspectos, estudando-os durante uma semana.

Ex.: « $1^{\text {a }}$ semana: comunicação verbal» «2 $2^{a}$ semana: comunicação não-verbal» « $3^{\text {a }}$ semana: terapêutica da dor» " $4^{\text {a }}$ semana: apoio à família»

- NÃO EXISTEM SINTOMAS INCONTROLÁVEIS, EXISTEM SINTOMAS MAL AVALIADOS

Um sintoma pode ter mais do que um factor na sua génese, portanto o modelo simples: 1 causa $\rightarrow 1$ efeito pode revelar-se redutor na avaliação de muitos quadros clínicos, devendo, por isso, considerar-se sempre que possível a possibilidade de que várias causas $\rightarrow$ efeito(s). 
O desafio coloca-se, principalmente ao jovem estudante, na tarefa de avaliação sintomática para uma intervenção terapêutica mais eficaz.

Para tal, devem seguir-se alguns princípios gerais como: avaliação da causa mais provável; explicação da causa dos sintomas; antecipação do tratamento antes da exacerbação de futuras queixas; adopção de terapêuticas farmacológicas e não-farmacológicas e monitorização dos sintomas. ${ }^{9}$

A avaliação e monitorização sintomáticas podem ser feitas através de escalas. Existem inúmeras escalas de sintomas adequadas de forma variável a diferentes tipos de doentes. Cada estudante deve, pelo menos, conhecer e ser portador de duas escalas no bolso da sua bata: 1) ESAS (Escala de Avaliação de Sintomas de Edmonton) que utiliza uma classificação numérica para avaliar uma série de sintomas e 2) Escala visual de dor.

- PRESTAR ATENÇÃO A PORMENORES E ALTERÁ-LOS

Os Cuidados Paliativos canalizam a sua actuação numa resposta adequada às necessidades dos doentes. Na intervenção activa do sofrimento associado à doença avançada e/ou terminal os intervenientes neste processo devem conhecer os factores centrais na qualidade de vida dos doentes avançados: ${ }^{15}$

- Controlo adequado da dor e outros sintomas;

- Evitar prolongamento inadequado da agonia;

- Manter o controlo;

- Evitar ser um fardo para quem o rodeia;

- Fortalecer as relações familiares;

Assim, ciente destes factores, o estudante deve estar preparado para identificar e alterar situações que envolvam a devolução da autonomia (por mínima que seja para o doente), da auto-estima, da dignidade e da privacidade.

\section{- ACEITAR QUE «SER MÉDICO» É TAMBÉM: AJUDAR QUEM MORRE, AJUDAR A VIDA DE QUEM FICA}

As doenças crónicas, progressivas e incuráveis, seja elas de etiologia neoplásica, doenças de órgão ou outras, estão associadas a períodos de perda e processos de luto patológico que se reflectem com expressão variável nos familiares, cuidadores e profissionais de saúde. A compreensão do sofrimento que a perda gera e a disponibilização de apoio, mesmo que momentâneo, pode ser uma aptidão simples a ser aprendida pelo es- tudante e conduzirá, certamente, a que esta fase da vida de quem fica seja partilhada e menos dolorosa.

- QUANDO NÃO EXISTE CURA, HÁMUITO ASER FEITO

Os Cuidados Paliativos não são o último recurso depois de um insucesso.

São um conhecimento médico, científico, rigoroso e humanizado a exercer atempadamente.

\section{CONCLUSÃO}

Confrontadas com a nova realidade da emergência das doenças crónicas, progressivas e incuráveis, as sociedades aperfeiçoam esforços na tentativa de implementação de um conhecimento médico rigoroso e humanizado, baseado em evidência científica.

Tal esforço deve penetrar na estrutura académica, iniciando lentamente a formação específica pré-graduada no âmbito de Cuidados Paliativos para que, idealmente no futuro, faça parte obrigatória do currículo médico.

Até que tal aconteça, serve esta reflexão «por detrás dos olhos de um estudante» para tentar alertar para o défice de formação na área dos Cuidados Paliativos, fornecendo algumas formas para que o estudante ultrapasse as insuficiências formativas académicas.

O estudante de Medicina deve especial atenção à perseverança e determinação, apoiadas num conhecimento sólido baseado em formação e evidência científica.

Deve ser enquanto estudantes que todos o médicos ganham a certeza de que ajudando os doentes no seu sofrimento global, ultrapassam o seu papel de curadores de doenças, para recuperarem a herança de curadores/cuidadores da pessoa.

\section{REFERÊNCIAS BIBLIOGRÁFICAS}

1. Vitorino RM, Jolie C, Mckimm J. O licenciado médico em Portugal: Core Graduates Learning Outcome Project. Lisboa: Faculdade de Medicina de Lisboa; 2005.

2. European Association for Palliative Care. Disponível em: http://www.eapcnet.org/ [acedido em 30/04/2009].

3. Toulmin S. On the nature of the physician's understanding. J Med Philos 1976 Mar; 1 (1): 32-50.

4. Egnew TR. The meaning of healing: transcending suffering. Ann Fam Med 2005 May-Jun; 3 (3): 255-62.

5. Glare P, Virik K. Can we do better in end-of-life care? The mixed management model and palliative care. Med J Aust 2001 Nov 19; 175 (10): 530-3.

6. Billings JA, Block S. Palliative care in undergraduate medical education: 
status report and future directions. JAMA 1997 Sep 3; 278 (9): 733-8.

7. Dowling $S$, Broomfield D. Undergraduate teaching in palliative care in Irish medical schools: a questionnaire survey. Med Educ 2003 May; 37 (5): 455-7.

8. Twycross R. Cuidados Paliativos. $2^{\text {a }}$ ed. Lisboa: Climepsi; 2003.

9. Barbosa A, Neto IG, editores. Manual de Cuidados Paliativos. $1^{\text {a }}$ ed. Lisboa: Faculdade de Medicina de Lisboa; 2006.

10. Doyle D, Hanks GW, Cherney N, Calman K, editors. Oxford Textbook of Palliative Medicine. 3rd ed. London: Oxford University Press; 2004.

11. Albom M. Às Terças com Morrie. Porto: Público; 2003.

12. Schwartz MS. Morrie in his own words. New York:Walker and Company; 1999.

13. Schmitt EE. Óscar e a Senhora Cor-de-Rosa. Lisboa: Âmbar; 2007.
14. Pausch R. A Última Aula. Lisboa: Editorial Presença; 2008.

15. Singer PA, Martin DK, Kelner M. Quality end-of-life care: patients' perspectives. JAMA 1999 Jan 13; 281 (2): 163-8.

\section{ENDEREÇO PARA CORRESPONDÊNCIA}

Miguel Julião

Rua Pedro Hispano, 19, $7^{\circ}$ esquerdo

2790-250 Carnaxide

E-mail: migueljuliao@gmail.com

Recebido em 26/08/2008

Aceite para publicação em 06/03/2009

\section{ABSTRACT}

Palliative Medicine is a medical, scientific, rigorous and humanized knowledge that treats patients and their families with chronic, progressive and incurable diseases.

During his academic curriculum, the medical student centralizes his strengths in the physiologic process of particular diseases, diagnosis and treatment. The undergraduate medical curriculum doesn' $t$ contemplate the principles of Palliative Care and rarely defines the individual on a holistic perspective. Young medical students are trained as biomedical scientists, doctors that cure the disease, rather than healers of the individual as a whole.

It's desirable that the undergraduate Palliative Care teaching slowly penetrates the academic structure, ideally becoming an obligatory part of the curriculum.

Through his personal experience, the author intends to give some orientations to young medical students to overcome the difficulties found when trying to transmit and practice the principles of Palliative Care within medical teams that have deficient knowledge on this specific area.

Keywords: Palliative Care; Undergraduate Medical Teaching. 